\title{
Carcinosarcoma of the breast: report of a case and review of the literature
}

\author{
Alexandra Varlatzidou • Aikaterini Mastoraki • \\ Maria Kefala • Panagiotis Safioleas • \\ George Sakorafas • Michael Safioleas
}

Received: 17 July 2009 /Accepted: 6 August 2009/Published online: 3 September 2009

(C) Springer-Verlag 2009

\begin{abstract}
Carcinosarcoma (CS) of the breast is a rare, poorly differentiated, malignant tumor which represents less than $0.1 \%$ of all breast cancers. It is strictly defined as a mixed, biphasic neoplasm consisting of a carcinomatous component combined with a malignant nonepithelial element of mesenchymal origin. We present a female patient with breast CS. Relevant literature is briefly reviewed. A 65 -year-oldwoman was admitted for the management of a palpable mass of the left breast. Mammography depicted a high-density mass with marginal irregularity and pleomorphic calcification. Subsequent excisional biopsy revealed a biphasic metaplastic breast carcinoma, and the patient underwent a left-modified radical mastectomy. The final histopathologic diagnosis was defined as a metaplastic breast carcinoma of CS type, with a squamous cell epithelial component of intermediate and mainly low-grade differentiation. Recent case series describe $\mathrm{CS}$ as an uncommon lesion demonstrating an intimate admixture of adenocarcinoma and sarcoma. Current data represent further evidence of the dedifferentiation theory in the origin of CS as it runs a very aggressive course and bears an ominous prognosis. The tumor is characterized by intermingled carcinomatous and sarcomatous areas resembling leaf-like structures of phyllodes pattern. Associated cumulative 5 -year survival rate is estimated at $49 \%$.
\end{abstract}

\footnotetext{
A. Varlatzidou $\cdot$ A. Mastoraki $(\bowtie) \cdot$ M. Kefala $\cdot$ P. Safioleas

G. Sakorafas · M. Safioleas

4th Department of Surgery, Athens University, Medical School, ATTIKON University Hospital,

1 Rimini str.,

12462 Chaidari, Athens, Greece

e-mail: dr_kamast@yahoo.gr

Present address:

A. Mastoraki

29 Irodotou str.

15122 Maroussi, Athens, Greece
}

Keywords Breast carcinosarcoma · Biphasic tumors · Therapeutic approach $\cdot$ Literature review

\section{Introduction}

Carcinosarcoma (CS) of the breast is a rare, poorly differentiated, malignant tumor which represents less than $0.1 \%$ of all breast cancers [1]. It is strictly defined as a mixed, biphasic neoplasm consisting of a carcinomatous component combined with a malignant nonepithelial element of mesenchymal origin without an intermediate transition zone [2]. Though the exact histogenesis of CS remains controversial, recent studies indicate that it is a variety of metaplastic mammary carcinoma with multipotential myoepithelial cell origin [3]. The term "monophasic sarcomatoid carcinoma" is only initiated in case of identifying epithelial component at electron microscopy or immunohistochemical analysis. Biphasic tumors are intensively debated in the literature. Several terms such as "biphasic sarcomatoid carcinoma" and "carcinosarcoma" have occasionally been used. Increasing evidence suggests that similar neoplastic development occurs through a malignant transformation of myoepithelial cells or myofibroblastic metaplasia of malignant epithelial cells $[4,5]$. Clinically, CS presents as a tumor of variable size and may appear in such diverse locations besides the breast such as the uterus, thyroid, lung, colon, esophagus, prostate, and pancreas $[6,7]$. Differential diagnosis includes spindle cell carcinoma, malignant phyllodes tumor, and stromal sarcoma. Recent surveys suggest that CS behaves biologically different from conventional carcinoma of the breast as sarcomatoid characteristics dominate the clinical course [8].

We present a female patient with breast CS. Relevant literature is briefly reviewed. Clinical features, histogenesis, 
morphological, and immunochemical findings are discussed, while the role of current diagnostic and therapeutic management of this aggressive neoplasm is emphasized.

\section{Case report}

A 65-year-old-woman was admitted to our institution for the management of a palpable mass of the left breast. The lesion was discovered by the patient through palpation a year ago. Past medical history was irrelevant, and she was on no medication. Interestingly, both her grandmother and daughter suffered from breast cancer, while her father died from metastatic cancer of unknown origin. She also referred a noncontributory personal obstetric history. On clinical examination, an irregularly shaped, relatively mobile mass measuring approximately $2 \times 2 \mathrm{~cm}$ was palpable in the upper outer quadrant of the left breast. The mass was not fixed to surrounding tissues (skin and pectoral muscle). Regional lymphadenopathy was not detected. Mammography depicted a high-density mass with marginal irregularity and pleomorphic calcification. Subsequent excisional biopsy revealed a biphasic metaplastic breast carcinoma, and the patient underwent a left-modified radical mastectomy (MRM) combined with axillary lymph node dissection. Core needle biopsy was rejected in order to obtain adequate tissue to detect potential malignancy and confirm the diagnosis. Furthermore, sentinel node biopsy is usually avoided in our institution in a routine basis in an attempt to reassure a radical and safe surgical approach in each case of neoplastic lesion of the breast.

Macroscopically, the cut surface of the tumor seemed elastic and soft in consistency with solid and lobulated features. Maximal tumor diameter was estimated approximately at $2.5 \mathrm{~cm}$ with primarily white and less brownish

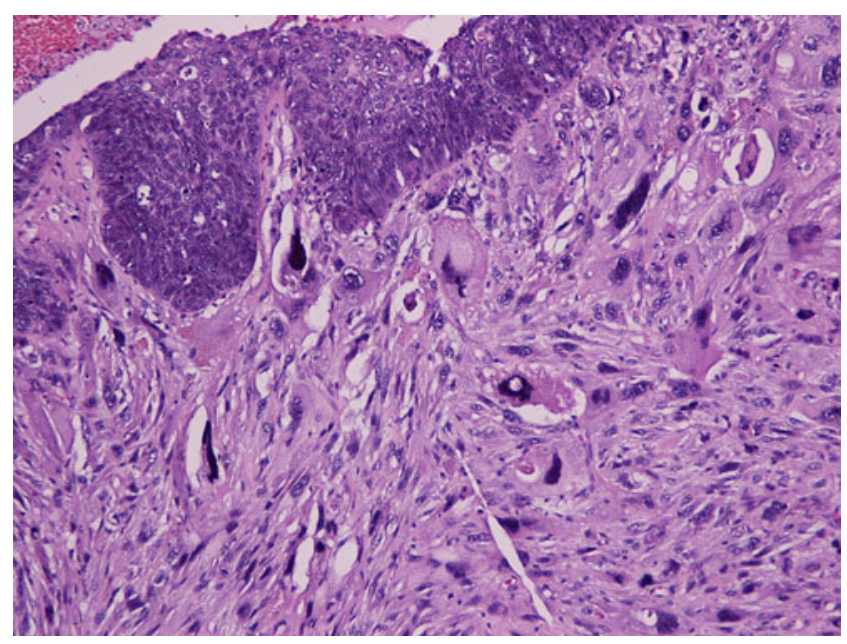

Fig. 1 Microscopical analysis of the tumor consisted of both carcinomatous and sarcomatous elements $(\mathrm{H}$ and $\mathrm{E}$ stain $\times 10)$

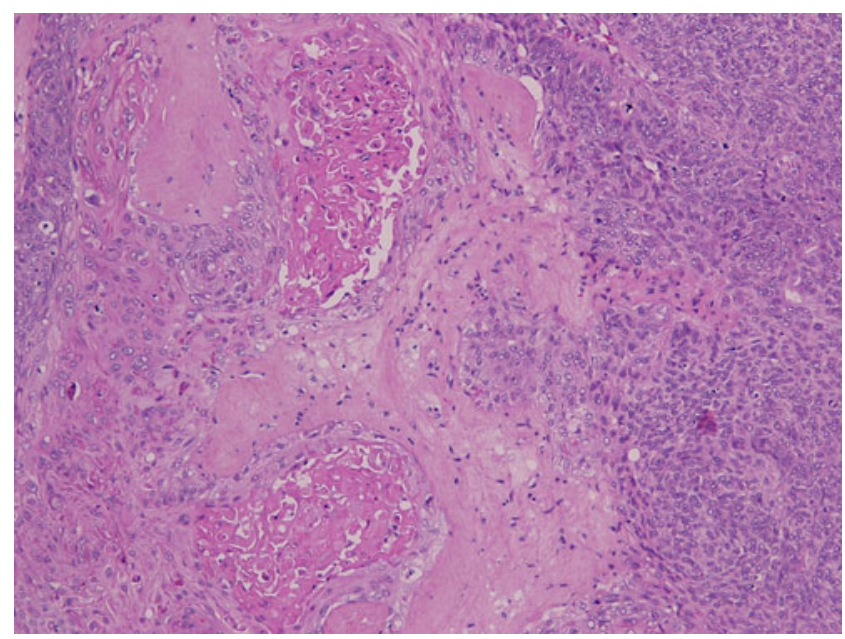

Fig. 2 The epithelial part of the tumor possessed ductal, keratin producing features of low-grade malignancy $(\mathrm{H}$ and $\mathrm{E}$ stain $\times 10)$

appearance. The skin was not infiltrated by the mass. Tissue sample was fixed in $10 \%$ formalin. Paraffin sections were prepared and stained with hematoxyllin and eosin. Additional immunostaining according to the avidin-biotin peroxidase method with anticytokeratin antibodies such as AE1/AE3, CK34ßE12, CK7, CKMNF16, antivimentin, antihuman smooth muscle actin (SMA), antidesmin, and anti-p63 antibody was accomplished.

Microscopically, the tumor consisted of both carcinomatous and sarcomatous elements (Fig. 1). The epithelial component was dominant, while mesenchymal cellular structure was evaluated less than $10 \%$. The carcinomatous part of the tumor possessed ductal, keratin producing features of low-grade malignancy (Fig. 2). The sarcomatous element consisted of pleomorphic spindle cells with giant nuclei and dispersed chromatin. These two components interlocked each other with distinct transition areas. Central necrosis and surrounding tissue infiltration was apparent. The tumor presented intense nucleus pleomorphism and marked mitotic activity accounting of 25/high power field (HPF) in the carcinomatous structure and 8/HPF in the sarcomatous one. Concomitant vascular invasion or metastasis to 22 removed lymph nodes was not observed.

Hormonal receptor assay in both the carcinomatous and sarcomatous component excluded estrogen and progesterone receptors' activity $(0 \%)$. Nucleus p53 stain proved intensively positive $(80 \%)$, Ki67 weakly positive, and c-erbB2 negative $(0 \%)$. Immunohistochemical staining demonstrated positive reaction in the epithelial element for cytokeratins CK34ßE12, CK7, AE1/AE3, CKMNF16, p63, and vimentin (Fig. 3). Further histochemical analysis of specific markers identified periodic acid-Schiff positivity. Sarcomatous cells proved positive for vimentin and negative for desmin, SMA, p63, and cytokeratins AE1/ 


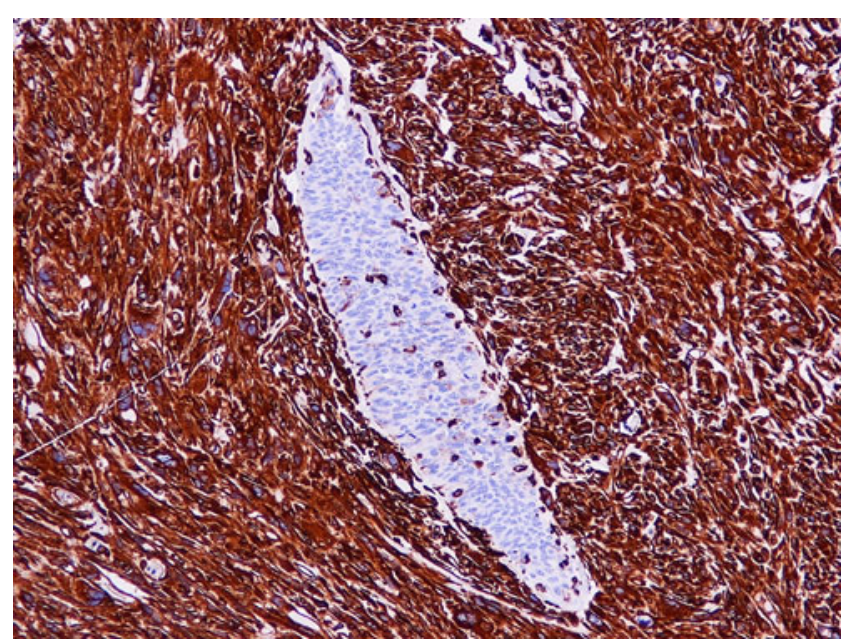

Fig. 3 Immunohistochemical staining proved vimentin positivity of both carcinomatous and sarcomatous components (vimentin stain $x 10)$

AE3, CKMNF16, CK34 $\beta$ E12, and CK7. Additionally, low-grade ductal carcinoma in situ was noticed in the rest of the mammary gland. The final histopathologic diagnosis was defined as a metaplastic breast carcinoma of CS type, with a squamous cell epithelial component of intermediate and mainly low-grade differentiation. The patient underwent systemic screening for distant metastasis, which was negative. Secondary hematogenous infiltrations were not defined. She had an uneventful recovery, and she was transferred to the Department of Oncology. She was administered 12 cycles of a combination of cyclophosphamide and epirubicin as adjuvant chemotherapy.

\section{Discussion}

Breast CS is extremely rare and accounts of less than $0.1 \%$ of all retrospectively diagnosed breast cancers. Histogenesis of the tumor remains controversial, while increasing data suggesting myoepithelial cell origin with potential biphasic differentiation have been reported [9]. Therefore, the tumor should be distinguished from other types of metaplastic carcinoma, including, spindle cell carcinoma, carcinoma with cartilaginous or osseous metaplasia, matrix producing carcinoma, fibrosarcoma, and various types of mesenchymal neoplastic disorders [2, 10]. Recent case series describe $\mathrm{CS}$ as an uncommon lesion demonstrating an intimate admixture of adenocarcinoma and sarcoma [11]. Current data represent further evidence of the dedifferentiation theory in the origin of CS as it runs a very aggressive course and bears an ominous prognosis. Associated cumulative 5 -year survival rate is estimated $49 \%$.

CSs are commonly described in various organs and anatomic sites including urinary bladder, uterus, liver, gastrointestinal tract, lungs, head, and neck [12-14]. Relevant clinicopathological and immunohistochemical features are debated in the literature. On the basis of case reports, primary heterotopous CS of the urinary bladder proved a highly malignant, rapidly developing neoplastic category occurring predominantly in elderly males, and diagnosed at an advanced stage [6]. Isolated cases of prostatic or cutaneous CS suffering from parallel widespread skeletal metastases have also been mentioned [15]. These included a papillary growth pattern combined with liposarcomatous differentiation [16]. Recent surveys document that in case of ovarian CS clonal loss of the BRCA2 allele, as well as, the same somatic mutation of the TP53 gene was evident in both cellular histologic components [17]. These data indicate that hereditary ovarian and breast CS may result from specific mutations that reassure common origin of both histologic elements from progenitor cell. A case of CS of the uterus with melanocytic differentiation has recently been published [18]. In case of esophageal CS differences in immunohistochemical and genetic clonalities suggest that the tumor composed of squamous cell carcinoma and leiomyosarcoma derived separately from epithelial and mesenchymal precursors $[19,20]$. Few cases have been observed in the small intestine, anorectal junction, liver, and pancreas [21, 22]. The most unique feature of colonic CS is its chondro and osteosarcomatous differentiation. Finally, a primary mediastinal CS has been discussed in the literature [23].

$\mathrm{CS}$ of the breast is an uncommon neoplastic lesion consisting of intraductal or infiltrating carcinoma contiguous or subtly merged with a highly cellular, mitotically active pleomorphic spindle cell stroma (sarcoma). Usually, demarcation between the carcinomatous and sarcomatous components is unclear in all light microscopic fields. The tumor is characterized by intermingled carcinomatous and sarcomatous areas resembling leaf-like structures of phyllodes pattern. Immunohistochemical staining shows positivity of the epithelial part for specific membrane antigens (EMA), cytokeratins, DF3 and p63. Further investigation of sarcomatous cells reveals positive expression of smoothmuscle actin (SMA), desmin, neuron-specific enolase (NSE), PGP 9.5, myoglobin and p53. Both components proved vimentin positive. In our case immunohistochemical examination demonstrated positive reaction in the epithelial element for cytokeratins CK34ßE12, CK7, AE1/AE3, CKMNF16, p63, and vimentin. Sarcomatous cells proved positive for vimentin and negative for desmin, SMA, p63, and cytokeratins AE1/AE3, CKMNF16, CK34 $\beta$ E12, and CK7 [24].

Treatment strategies for CS resemble those of typical breast cancer, including MRM with axillary dissection. Nevertheless, several differences have been reported between breast CSs and conventional adenocarcinomas [25]. 
Axillary lymph nodes are less frequently involved, and associated infiltration is not regarded as a strong predictor of the outcome. Hematogenous metastases are also frequent, with pleural and pulmonary metastasis being more common than skeletal, liver, or brain metastasis. Although data interpretation is restricted by retrospective and uncontrolled nature of the results, it appears that the administration of conventional radiotherapy may not adequately reduce the incidence of local recurrences for patients submitted to breast-conserving surgery. Recent studies suggest that MRM should be considered as a treatment of choice for each case of breast CS, particularly for patients with $\mathrm{T} 2$ and higher stage disease. Consequently, the optimization of locoregional control is critical [26]. Necessity of newly discovered radiation approaches and possibly concomitant radiosensitizing agents is widely accepted. In our case, a MRM was performed. Additional tumor metastases by the lymphogenous route were excluded.

Although patient numbers are restricted and interpretation limited by cross-trial comparisons, relevant prognosis of similar patients suffering from breast CS treated with adjuvant chemotherapy is equal to untreated cases' surveillance. Moreover, the activity of primary chemotherapy is certainly decreased in comparison with conventional adenocarcinoma, especially when one considers that highgrade hormone receptor-negative carcinomas are particularly sensitive to primary anthracycline/taxane-based therapeutic combination. Interestingly, the majority of breast CS patients treated with adjuvant sarcoma-type chemotherapy remain relapse free [27]. Even though limited series preclude definite statistical assessment, the outcome of cyclophosphamide, methotrexate, and 5-fluorouracil (CMF)-treated breast CS patients is poor compared with those receiving adjuvant anthracyclines, suggesting that CMF is less effective than anthracyclin-based therapeutic intervention in breast CS. In our case a combination of cyclophosphamide and epirubicin was administered as adjuvant chemotherapy 20 days after complete surgical recovery.

Conflict of interest There is no actual or potential conflict of interest in relation to this article.

\section{References}

1. Hennessy BT, Giordano S, Broglio K, Duan Z, Trent J, Buchholz TA et al (2006) Biphasic metaplastic sarcomatoid carcinoma of the breast. Annals of Oncology 17:605

2. Wick MR, Swanson PE (1993) Carcinosarcomas: current perspectives and an historical review of nosological concepts. Semin Diagn Pathol 10:118
3. Tokudome N, Sakamoto G, Sakai T, Sarumaru S, Okuyama N, Hori $\mathrm{F}$ et al (2005) A case of carcinosarcoma of the breast. Breast Cancer 12:149

4. Gogas J, Kouskos E, Markopoulos C, Mantas D, Antonopoulou Z, Kontzoglou K et al (2003) Carcinosarcoma of the breast: report of two cases. Eur J Gynaecol Oncol 24:93

5. Wada H, Enomoto T, Tsujimoto M, Nomura T, Murata Y, Shroyer KR (1998) Carcinosarcoma of the breast: molecular-biological study for analysis of histogenesis. Human Pathol 29:1324

6. Perret L, Chaubert P, Hessler D, Guillou L (1998) Primary heterologous carcinosarcoma (metaplastic carcinoma) of the urinary bladder: a clinicopathologic, immunohistochemical, and ultrastructural analysis of eight cases and a review of the literature. Cancer 82:1535

7. Tsekeris P, Dimou S (2000) Synchronous endometrial carcinosarcoma and breast carcinoma: a case report. Eur J Gynaecol Oncol 21:309

8. Cornette J, Tjalma WA, Buytaert P (2005) Biphasic sarcomatoid carcinoma or carcinosarcoma of the breast: prognosis and therapy. Eur J Gynaecol Oncol 26:514

9. Padmanabhan V, Dahlstrom JE, Chong GC, Bennett G (1997) Phyllodes tumor with lobular carcinoma in situ and liposarcomatous stroma. Pathology 29:224

10. Ferrara G (1995) Sarcomatoid carcinoma of the breast: pathology of four cases. Breast Dis 8:283

11. Balercia G, Bhan AK, Dickersin GR (1995) Sarcomatoid carcinoma: an ultrastructural stydy with light microscopic and immunohistochemical correlation of 10 cases from various anatomic sites. Ultrastructural Pathol 19:249

12. Higashi M, Takao S, Sato E (1999) Sarcomatoid carcinoma of the pancreas: a case report with immunohistochemical study. Pathol Int 49:453

13. Poblet E, Gomez-Tierno A, Alfaro L (2000) Prostatic carcinosarcoma: a case originating in a previous ductal adenocarcinoma of the prostate. Pathol Res Pract 196:569

14. Winfield HL, Rosenberg AS, Antonescu CR, Weil M, Wang AR (2003) Monophasic sarcomatoid carcinoma of the scalp: a case mimicking inflammatory myofibroblastic tumor and a review of cutaneous spindle cell tumors with myofibroblastic differentiation. J Cutan Pathol 30:393

15. Ram R, Saadat P, Peng D, Vadmal M (2005) Case report and literature review: primary cutaneous carcinosarcoma. Ann Clin Lab Sci 35:189

16. Lindboe CF, Klem KH (2006) Carcinosarcoma of the prostate. APMIS 114:153

17. Sonoda Y, Saigo PE, Federici MG, Boyd J (2000) Carcinosarcoma of the ovary in a patient with a germline BRCA2 mutation: evidence for monoclonal origin. Gynecol Oncol 76:226

18. Kajo K, Zubor P, Spacek J, Ryska A (2007) Carcinosarcoma of the uterus with melanocytic differentiation. Pathol Res Pract 203:753

19. Iwaya T, Maesawa C, Tamura G, Sato N, Ikeda K, Sasaki A et al (1997) Esophageal carcinosarcoma: a genetic analysis. Gastroenterology 113:973

20. Iwaya T, Maesawa C, Uesugi N, Kimura T, Ogasawara S, Ikeda K (2006) True carcinosarcoma of the esophagus. Dis Esophagus 19:48

21. Aramendi T, Fernandez-Acenero MJ, Villanueva MC (2003) Carcinosarcoma of the colon: report of a rare tumor. Pathol Res Pract 199:345

22. Di Vizio D, Insabato L, Conzo G, Zafonte BT, Ferrara G, Pettinato G (2001) Sarcomatoid carcinoma of the colon: a case report with literature review. Tumori 87:431

23. Bayram AS, Ozdemir B, Aydiner F, Gullulu S (2004) Carcinosarcoma as a primary mediastinal tumor. Interact Cardiovasc Thorac Surg 3:11 
24. Nielsen TO, Hsu FD, Jensen K (2004) Immunohistochemical and clinical characterization of the basal-like subtype of invasive breast carcinoma. Clin Cancer Res 10:5367

25. Gutman H, Pollock RE, Janjan NA, Johnston DA (1995) Biologic distinctions and therapeutic implications of sarcomatoid metaplasia of epithelial carcinoma of the breast. J Am Coll Surg 180:193
26. Foscini MP, Dina RE, Eusebi V (1993) Sarcomatoid neoplasms of the breast: proposed definitions for biphasic and monophasic sarcomatoid mammary carcinomas. Semin Diagn Pathol 10:128

27. Husband JE, Schwartz LH, Spencer J (2004) Evaluation of the response to treatment of solid tumours- a consensus statement of the International Cancer Imaging Society. Br J Cancer 90:2256 